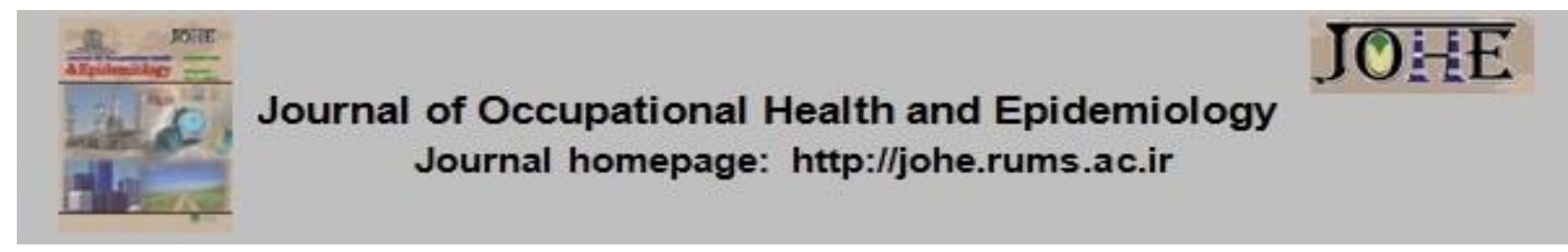

\title{
Diabetic Ketoacidosis and COVID-19: Two Case Reports
}

\author{
Mehdi Kafi $^{1}$, Maryam Karimi Fard ${ }^{2}$, Sima Amiorroaya Yamini ${ }^{3}$ \\ 1. Student Research Committee, Rafsanjan University of Medical Sciences, Rafsanjan, Iran. \\ 2. Assistant Prof., Non-Communicable Diseases Research Center, Rafsanjan University of Medical Sciences, Rafsanjan, Iran. \\ 3. Associate Prof., Dept. of Engineering and Mathematics, Sheffield Hallam University, Sheffield S1 1WB, UK.
}

Citation: Kafi M, Karimi Fard M, Amiorroaya Yamini S. Diabetic Ketoacidosis and COVID-19:

Two Case Reports. JOHE 2021; 10(1):12-6.

\section{Article Info}

* Corresponding author: Maryam Karimi Fard,

E-mail:

m.karimifard@rums.ac.ir

\section{Article history \\ Received: Dec 2020 \\ Accepted: Feb 2021}

10.29252/johe.10.1.12

Print ISSN: 2251-8096 Online ISSN: 2252-0902

Peer review under
responsibility of Journal of
Occupational Health and
Epidemiology

\begin{abstract}
Background: COVID-19 is a viral infection that causes pneumonia with dyspnea, cough, and fever. Its outcomes are more severe in patients with diabetes, hypertension, and other disorders than in healthy people. New-onset diabetes and diabetic ketoacidosis (DKA) have been reported as the complications of COVID-19 in several studies. It seems that the prevalence of DKA due to Covid-19 is increasing. Therefore, two cases of individuals with DKA due to COVID-19 are presented in this study to inform other researchers about the details of this phenomenon.

Material and Methods: Demographic characteristics, medical histories, physical examinations, laboratory investigations, real-time RT-PCR tests, computed tomography (CT) imaging studies, given treatments, clinical courses, and management outcomes were documented prospectively.

Results: In the present experience, the manifestation of COVID-19 disease in the second case with a history of diabetes was more severe than in the first case. In the second case, with underlying diabetes and COVID-19, the DKA manifestation was associated with consciousness loss, severe restlessness, and respiratory distress; however, in the first case, with COVID-19 without diabetes, the DKA manifestation was associated with anorexia, weight loss, and lack of respiratory distress, which were milder symptoms than the second case.

Conclusion: Due to the high prevalence of diabetes mellitus and COVID-19 in Iran, it is recommended to raise awareness of DKA symptoms among health professionals.
\end{abstract}

Keywords: Diabetes Complications, Diabetic Ketoacidosis, Covid-19

\section{Introduction}

COVID-19 was first diagnosed with pneumonia in December 2019 in Wuhan, China [1]. Patients can be asymptomatic or exhibit a mild upper respiratory failure to severe pneumonia with respiratory failure and even death [2,3]. Symptoms, including fever, cough, dyspnea, muscle aches, nausea, vomiting, headache, sore throat, chest pain, and diarrhea, can also vary from patient to patient [4]. On the other hand, the disease mortality rate is directly related to age, underlying diseases, such as diabetes, cardiovascular disorders, and cancer [4]. DKA, which can be fatal, is a disorder characterized by metabolic acidosis $(\mathrm{pH}<7.35$ or serum bicarbonate $\leq 15 \mathrm{mmol} / \mathrm{L})$, ketonemia or ketonuria, and hyperglycemia (blood glucose $\geq 250$ $\mathrm{mg} / \mathrm{dL}$ ) [5]. It occurs in patients with diabetes, especially type 1 , due to factors such as missing the insulin doses and significant physical or emotional stress, including infections [6]; it can also be present with sudden metabolic decompensation [7]. During the pandemic, several new-onset diabetes with DKA have been reported in COVID-19 patients [8, 9]. Here, the clinical and paraclinical features of two Covid-19 patients, one with a history of type 2 diabetes (T2D) and one with no history of diabetes eventually hospitalized due to DKA, are investigated. It seems that the 
prevalence of DKA due to Covid-19 is increasing; thus, in the present research, two cases of individuals with DKA due to COVID-19 are presented to raise awareness of DKA symptoms among health professionals at the time of the Covid-19 pandemic.

\section{Materials and Methods}

Demographic characteristics, detailed drug and medical histories, physical examinations, laboratory investigations, including Hematology, Biochemistry, Urine analysis, and Arterial Blood Gases (ABG), as well as real-time RT-PCR tests, computed tomography (CT) imaging studies for Covid-19, given treatments, clinical courses, and management outcomes, were documented prospectively. Informed consent was obtained from both patients for the study. Diabetic ketoacidosis was defined as plasma glucose $>250 \mathrm{mg} / \mathrm{dL}$, a positive test for urine ketones, and arterial $\mathrm{pH}<$ 7.35 .

Written informed consent was obtained from the patients. Since this was a case report but not a clinical study, ethics approval was unnecessary.

\section{Results}

Case 1: The first patient was a 48-year-old male, an Iranian farmer, who presented to the emergency department of Aliebne Abitaleb General Hospital in Rafsanjan, Iran, with fever and dyspnea. Due to suspicion of contact with the Covid-19 patient, a chest CT scan and RT-PCR test were conducted. The ground-glass opacity was observed in the lower and upper lobes of the right lung and the lower lobe of the left lung, and atelectasis was observed in the lower lobe of the left lung and lingula in the chest CT scan, confirming lung involvement (Fig.1). The patient's RT-PCR was also tested positive, confirming the diagnosis of COVID-19. Therefore, the patient underwent outpatient treatment and self-isolation at home. During the treatment course, he was prescribed intravenous dexamethasone, $8 \mathrm{mg}$ daily for 10 days, subcutaneous interferon-beta injections (Recigen), $44 \mu \mathrm{g}$ for 5 days every other day, with liver function monitoring tests, and a dose of Neurobion ampoule. Twelve days later, the patient was presented to the emergency department with anorexia, severe weight loss of $17 \mathrm{~kg}(88 \mathrm{~kg}$ to $71 \mathrm{~kg})$, polyuria and polydipsia in the last two weeks, and severe weakness on the day of admission. He exhibited no respiratory symptoms and had no history of diabetes or any other diseases. He had no history of drinking alcohol and/or using drugs, and of close relatives, only his uncle had T2D. The patient had no drug allergy or addiction. At admission, he had a body temperature of 36 degrees, pulse rate of 141 beats/minute, respiratory rate of 18 breaths/minute, blood pressure of $100 / 70 \mathrm{mmHg}$, body mass index (BMI) of $21.91 \mathrm{~kg} / \mathrm{m} 2$ (height 180 $\mathrm{cm}$ ), and oxygen saturation of $93 \%$ by pulse oximetry on ambient air. On examination, the patient was alert and showed severe dehydration signs, including weakness, rapid pulse, dry tongue, and skin. Chest X-ray was normal. Laboratory evaluation was notable for DKA (Table 1), with a random blood glucose of $800 \mathrm{mg} / \mathrm{dl}$ and $\mathrm{HbA} 1 \mathrm{C}$ of $10.3 \%$. Metabolic acidosis (bicarbonate $=8.4$ ) was also detected by arterial blood gas (ABG). His urine dipstick test was positive for ketones. Due to a history of COVID-19, the patient underwent another chest CT scan, which came back clear. Therefore, the patient was admitted to the hospital with the diagnosis of new-onset diabetes with DKA. He was started on infusion regular insulin, aggressive intravenous hydration, electrolyte replacement, and supportive measures. The patient's DKA was treated after 16 hours. Finally, the patient was discharged after treatment with a subcutaneous insulin regimen.

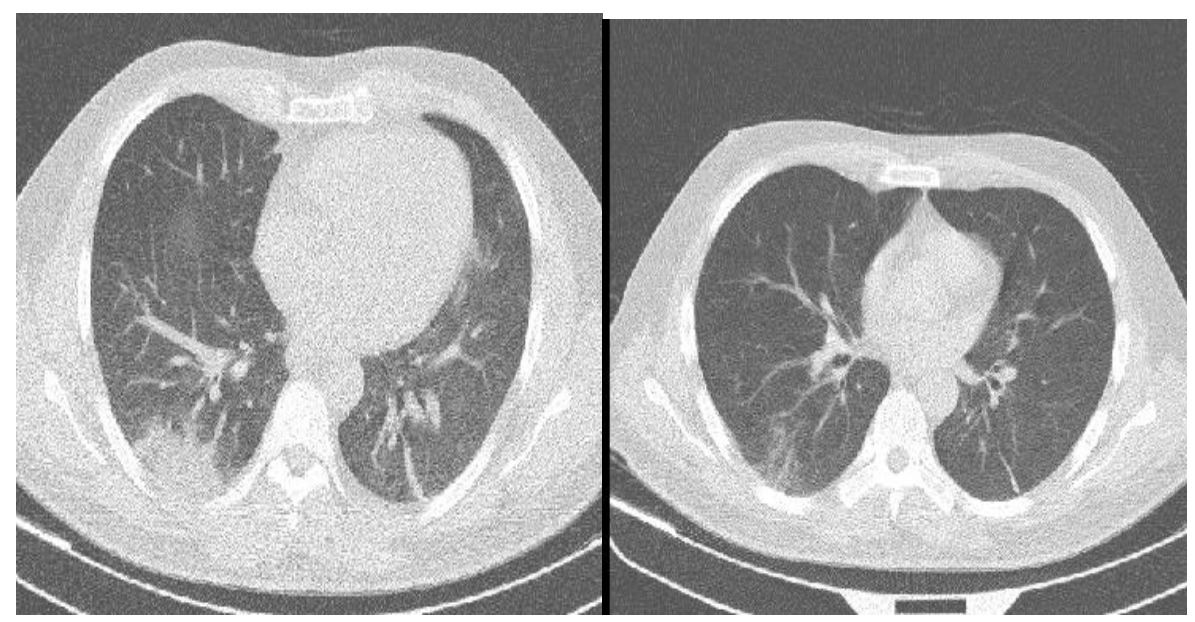

Fig.1. (a) Ground-glass opacity observed in the lower and upper lobes of the right lung and the lower lobe of the left lung (b) Atelectasis observed in the lower lobe of the left lung and lingual 
Table 1. Laboratory investigations of the two patients at admission

\begin{tabular}{|c|c|c|c|c|}
\hline Lab data & Patient & Normal range & Case 2 & Case 1 \\
\hline \multirow{7}{*}{ Hematology } & Hemoglobin $(\mathrm{g} / \mathrm{dl})$ & 18.9 & 11 & $14-18$ \\
\hline & White Blood Cell $(/ \mu \mathrm{L})$ & 11500 & 13200 & $4000-10000$ \\
\hline & Lymphocyte count $\left({ }^{*} 10^{3} / \mu \mathrm{L}\right)$ & 528 & 660 & $1000-4800$ \\
\hline & Red Blood Cell (Mil/ $\mu \mathrm{L})$ & 6.65 & 4.20 & $4.5-6.5$ \\
\hline & Erythrocyte Sedimentation Rate $(\mathrm{mm} / \mathrm{h})$ & 8 & 15 & $<50$ \\
\hline & C Reactive Protein & 10 & 8 & Up to 5 \\
\hline & Blood Glucose (mg/dl) & 800 & 549 & $65-100$ \\
\hline \multirow{15}{*}{ Biochemistry } & Creatinine $(\mathrm{mg} / \mathrm{dl})$ & 2.1 & 2 & $0.6-1.4$ \\
\hline & Urea $(\mathrm{mg} / \mathrm{dl})$ & 96 & 66 & $17-43$ \\
\hline & Calcium $(\mathrm{mg} / \mathrm{dl})$ & 8.8 & 12.1 & $8.4-10.2$ \\
\hline & Potassium (mEq/L) & 6.2 & 5.5 & $3.8-5$ \\
\hline & Phosphate (mg/dl) & 7.2 & 7.7 & $2.7-4.5$ \\
\hline & Sodium (mEg/l) & 152 & 147 & $132-142$ \\
\hline & Lactate Dehydrogenase (U/L) & 726 & 1200 & Up to530 \\
\hline & Aspartate Transaminase $(\mathrm{U} / \mathrm{L})$ & 51 & 500 & Up to 37 \\
\hline & Alanine Transaminase $(\mathrm{U} / \mathrm{L})$ & 69 & 292 & Up to 41 \\
\hline & Alkaline Phosphatase (U/L) & 210 & 280 & $80-306$ \\
\hline & Bilirubin total & 1.4 & 0.9 & $0.1-1.2$ \\
\hline & Bilirubin direct & 5.0 & 0.1 & $0.1-0.3$ \\
\hline & Creatine Phosphokinase (U/L) & 183 & 1000 & 24-195 \\
\hline & Hemoglobin $A_{1} C(\%)$ & 10.3 & 12 & Up to 5.5 \\
\hline & Troponin & - & - & - \\
\hline \multirow{4}{*}{ Urine } & $\mathrm{PH}$ & 5 & 6 & $4.5-8$ \\
\hline & Ketone & + & +++ & - \\
\hline & Protein & - & - & - \\
\hline & Glucose & - & ++ & - \\
\hline \multirow{3}{*}{$\begin{array}{l}\text { Arterial Blood } \\
\text { Gases (ABG) }\end{array}$} & $\mathrm{PH}$ & 7.2 & 6.9 & $7.25-7.35$ \\
\hline & $\mathrm{HCO}_{3}$ & 8.4 & 2 & $22-24$ \\
\hline & $\mathrm{O}_{2}$ saturation $(\%)$ & 93 & 94 & $95-100$ \\
\hline
\end{tabular}

Case 2: The second patient was a 60-year-old male, a manual worker from Afghanistan, who presented to the emergency department of Aliebne Abitaleb General Hospital in Rafsanjan with fever, restlessness, and respiratory distress with no need for intubation though. According to the patient's symptoms and confirmed exposure to the COVID19 patient, a chest CT scan and RT-PCR were conducted. The patient was diagnosed with T2D for a year and hypertension for three years. He had no known diabetes-related complications and no follow-up visit for controlling his blood glucose. The patient had no addiction or allergy to drugs. Bilateral opacity of ground glass was observed in radiographic lung opacities (Fig. 2), and due to a positive RT-PCR test, the diagnosis of severe COVID-19 was confirmed. Therefore, he was hospitalized and administered oxygen, a subcutaneous insulin regimen, dexamethasone (8 $\mathrm{mg}$ daily), ceftriaxone ( $1 \mathrm{~g}$ twice a day), and azithromycin with supportive care. After three days, the patient was discharged with personal consent. $\mathrm{He}$ was prescribed Tavanx (500 mg daily), Glucophage (500 mg twice a day), and losartan (25 $\mathrm{mg}$ daily). Two weeks later, the patient was presented to the emergency department again with a sudden consciousness loss, severe restlessness, and respiratory distress. At admission, the patient was hypotensive (70/50 $\mathrm{mmHg}$ ), tachycardia $(\mathrm{HR}=120)$, and afebrile (body temperature of 37 degrees). His respiratory rate was 20 breaths/minute, and he had a BMI of 19.6 $\mathrm{kg} / \mathrm{m} 2$ (height $182 \mathrm{~cm}$ and weight $65 \mathrm{~kg}$ ). The oxygen saturation was $94 \%$ on ambient air. On examination, the patient was severely dehydrated; however, other physical examinations were normal despite his confusion. The laboratory analysis (Table 1) showed a random blood glucose of 549 $\mathrm{mg} / \mathrm{dl}$ and $\mathrm{HbA} 1 \mathrm{C}$ of $12 \%$. Metabolic acidosis (bicarbonate=2) was also detected in the ABG. His dipstick urinalysis was positive for ketones (3+) with detected sugar. Due to the loss of consciousness, a brain CT scan was performed on him, which came back normal. Therefore, he was treated for DKA performing fluid bolus therapy by normal saline, replacing the sodium and free water deficit over 24 hours, administrating regular intravenous insulin, and broad-spectrum antibiotics, and sepsis workup. His DKA was improved after 30 hours, and he was discharged in good general condition. 
Fig.2. Bilateral opacity of ground glass

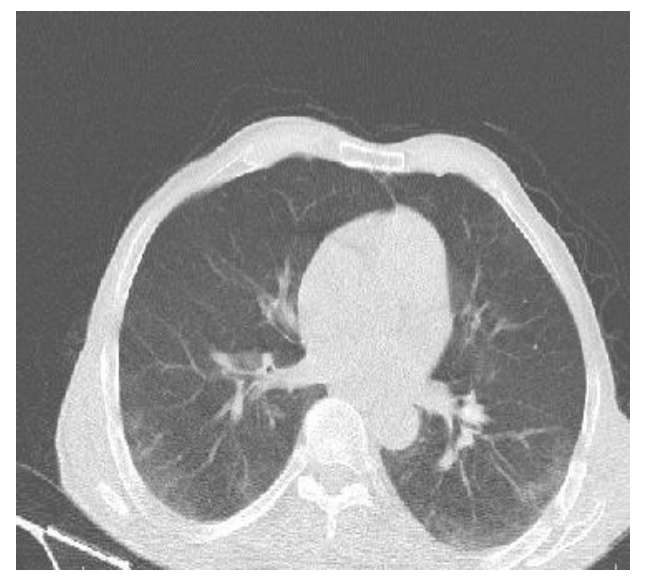

\section{Discussion}

In this study, two patients with a history of COVID19 were reported; both were admitted to the hospital with a diagnosis of DKA after a few days.

The first case had no history of diabetes mellitus, but the second one had. The role of some disorders, such as diabetes, obesity, and cardiovascular, has already been identified as an aggravating factor in infectious diseases, especially COVID-19 [10]. In the present experience, the manifestation of COVID-19 in the second case with a history of diabetes was more severe than in the first case. The relationship between various infections, such as measles, rubella, EBV, and CMV, with different types of diabetes, has been previously identified, proving that DKA, as a complication of diabetes, can be caused by infections [9]. On the other hand, COVID-19 can also cause new-onset diabetes with DKA in people with or without known diabetes [11]. In this condition, possible causes of DKA include dysfunction of beta cells in the pancreas by COVID-19 virus [8] and interactions between COVID-19 and the angiotensin aldosterone renin (RAAS) system, which increases insulin resistance [12]. The inflammatory and autoimmune properties of COVID-19 [13] and the role of cytokine storms that occur with the onset of infection and cause extrapulmonary manifestations are very important; cytokines include DPP4, IL-6, IL-1B, and TNF [1416]. In this study, $\mathrm{HbA} 1 \mathrm{C}$ level was elevated in both patients, with a higher level in the second and more severe DKA; however, the level of $\mathrm{HbA} 1 \mathrm{C}$ can be unreliable in some conditions, such as HIV infection; therefore, it may not also be reliable in patients with COVID-19 [17]. In addition, the severity of DKA is associated with its causes, which, in more than $50 \%$ of cases, is due to infections, having more severe manifestations in patients with underlying diabetes [18]. In the second case, with underlying diabetes and COVID-
19, the DKA manifestation was associated with consciousness loss, severe restlessness, and respiratory distress; however, in the first case, with COVID-19 without diabetes, the DKA manifestation was associated with was anorexia, weight loss, and lack of respiratory distress, which were milder symptoms than the second case. On the other hand, both patients of the current report were presented to the hospital with DKA following two weeks of treatment for their diagnosed COVID-19; therefore, the possible causes of DKA in these patients might be associated with the medications received for their treatment, including dexamethasone.

\section{Conclusion}

Due to the high prevalence of diabetes mellitus and COVID-19 in Iran, it is recommended to raise awareness of DKA symptoms among health professionals at the time of the pandemic, emphasizing the control of patients' blood glucose within the normal range, managing the dose of insulin in patients with diabetes, and advising patients to drink fluids and seek medical attention if dehydrated and/or exhibited persistent vomiting.

\section{Acknowledgement}

We thank the medical staff who participated in managing patients, especially the men's Internal ward of Aliebne Abitaleb Hospital in Rafsanjan.

Conflict of interest: None declared.

\section{References}

1. Phelan AL, Katz R, Gostin LO. The Novel Coronavirus Originating in Wuhan, China: Challenges for Global Health Governance. JAMA 2020; 323(8):709-10.

2. Wang D, Hu B, Hu C, Zhu F, Liu X, Zhang J, et al. Clinical Characteristics of 138 Hospitalized Patients with 2019 Novel Coronavirus-Infected 
Pneumonia in Wuhan, China. JAMA 2020; 323(11):1061-9.

3. Huang C, Wang Y, Li X, Ren L, Zhao J, Hu, Y et al. Clinical features of patients infected with 2019 novel coronavirus in Wuhan, China. Lancet 2020; 395(10223):497-506.

4. Yang W, Cao Q, Qin L, Wang X, Cheng Z, Pan $A$, et al. Clinical characteristics and imaging manifestations of the 2019 novel coronavirus disease (COVID-19): A multi-center study in Wenzhou city, Zhejiang, China. J Infection 2020; 80(4):388-93.

5. Benoit SR, Zhang Y, Geiss LS, Gregg EW, Albright A. Trends in Diabetic Ketoacidosis Hospitalizations and In-Hospital Mortality United States, 2000-2014. MMWR Morb Mortal Wkly Rep 2018; 67(12):362-5.

6. Gautam S. The Influence of COVID-19 on Air Quality in India: A Boon or Inutile. Bull Environ Contam Toxicol 2020; 104:724-6.

7. Plewa MC, Bryant $M$, King-Thiele R. Euglycemic Diabetic Ketoacidosis. Treasure Island (FL), Florida, United States: StatPearls Publishing; 2021.

8. Reddy PK, Kuchay MS, Mehta Y, Mishra SK. Diabetic ketoacidosis precipitated by COVID-19: A report of two cases and review of literature. Diabetes Metab Syndr 2020; 14(5):1459-62.

9. Palermo NE, Sadhu AR, McDonnell ME. Diabetic Ketoacidosis in COVID-19: Unique Concerns and Considerations. J Clin Endocrinol Metab 2020; 105(8):dgaa360.

10. Apicella M, Campopiano MC, Mantuano $M$, Mazoni L, Coppelli A, Del Prato S. COVID-19 in people with diabetes: understanding the reasons for worse outcomes. Lancet Diabetes Endocrinol 2020; 8(9):782-92.
11. Li J, Wang X, Chen J, Zuo X, Zhang H, Deng A. COVID-19 infection may cause ketosis and ketoacidosis. Diabetes Obes Metab 2020; 22(10):1935-41.

12. Bourgonje AR, Abdulle AE, Timens W, Hillebrands JL, Navis GJ, Gordijn SJ, et al. Angiotensin-converting enzyme-2 (ACE2), SARS-CoV-2 and pathophysiology of coronavirus disease 2019 (COVID-19). J Pathol 2020; 251(3):228-48.

13. Rodríguez $Y$, Novelli L, Rojas $M$, De Santis $M$, Acosta-Ampudia Y, Monsalve DM, et al. Autoinflammatory and autoimmune conditions at the crossroad of COVID-19. J Autoimmun 2020; $114: 102506$.

14. Manson JJ, Crooks C, Naja M, Ledlie A, Goulden B, Liddle T, et al. COVID-19associated hyperinflammation and escalation of patient care: a retrospective longitudinal cohort study. Lancet Rheumatol 2020; 2(10):e594-602.

15. Makdissi A, Ghanim H, Vora M, Green K, Abuaysheh S, Chaudhuri A, et al. Sitagliptin exerts an antinflammatory action. J Clin Endocrinol Metab 2012; 97(9):3333-41.

16. Pinheiro MM, Stoppa CL, Valduga CJ, Okuyama CE, Gorjão R, Pereira RM, et al. Sitagliptin inhibit human lymphocytes proliferation and Th1/Th17 differentiation in vitro. Eur J Pharm Sci 2017; 100:17-24.

17. American Diabetes Association. 2. Classification and Diagnosis of Diabetes: Standards of Medical Care in Diabetes-2020. Diabetes Care 2020; 43(Suppl 1):S14-31.

18. Nyenwe EA, Kitabchi AE. The evolution of diabetic ketoacidosis: An update of its etiology, pathogenesis and management. Metabolism 2016; 65(4):507-21. 